\title{
Computational Fluid Dynamics Simulations of Heat Transfer of Identical Chimneys in a Building
}

\author{
Mohamad Mehyo ${ }^{1}$, José J. Costa ${ }^{2}$ \\ ${ }^{1}$ Department of Mechanical Engineering, Ondokuz Mayis University \\ Körfez Mahallesi, 19 Mayıs Ünv. Kurupelit Kampüsü, 55105 Atakum, Samsun, Turkey \\ 15210438@stu.omu.edu.tr \\ ${ }^{2}$ ADAI-LAETA, Mechanical Engineering Department, University of Coimbra \\ Pátio das Escolas 3004-531, Coimbra, Portugal \\ jose.costa@dem.uc.pt
}

\begin{abstract}
In recent years, numerical simulation heat transfer became a widely used for the chimneys simulation with the natural boundary. This simulation has been performed using an iterative technique line-by-line, which is based on Thomas algorithm or TDMA (3) (tri-diagonal matrix algorithm) and using the computational fluid dynamics (CFD) Fortran 90 code. CFD has been used to study natural ventilation. The main advantages of the CFD approach are that it enables to analyze complex gases flow patterns for fireplaces of buildings and that it can be used to simulate the chimneys flow performance for a lot of imaginary buildings for design purposes. The results are included the figures of the temperature distribution, as well as heat flow lines and vectors. The overall heat loss through the chimney walls has reached for chimneys 1 and 4 to 2876 and $2871(\mathrm{~W} / \mathrm{m})$ respectively. These values have been generated by the postprocessing program (Tecplot 360). The comparative assessment of mesh sizes shows that the results are close to the ideal situation which say that a mesh independent solution is one which does not change with further mesh refinement.This study has demonstrated that CFD is a powerful tool capable of capturing the complex heat transfer process (with some assumptions) in gas-solid structures with satisfactory results and is a useful tool in the design of other structures reactors.
\end{abstract}

Keywords: CFD, Heat Transfer, Simulation, Chimneys.

\section{Introduction}

Computational Fluid Dynamics (CFD) is a theoretical method of scientific and engineering investigation, concerned with the development and application of a virtual video - camera like tool - a software which is used to analyze a fluid dynamics as well as heat and mass transfer problem; for a unified cause-and-effect study. Here, the software results in a fluid-dynamic a movie where each picture consists of a flow property (velocity, pressure, temperature, vorticity, and streamfunction). Each flow property can result in one movie. Thus, a large number of fluid dynamic movie can be generated for a scientific understanding and engineering related study of a particular fluid dynamics problem. Representation, as well as mathematical-modeling of flow, are needed to create a fluid-dynamic movie in CFD [5]. Applying the fundamental laws of mechanics to a fluid gives the governing equation for a fluid. Partial differential equations are discretized into a system of algebraic equations. And all algebraic equations are then solved numerically to render the solution field. This equation is nonlinear partial differential equations. It is not possible to solve this equation analytically for most engineering problems. However, it is possible to obtain approximate computer-based solutions to the governing equations for a variety of engineering problems. This is the subject matter of CFD.

CFD is useful in a wide variety of applications. One of this applications is a numerical analysis of heat transfer in enclosures of importance in thermal science [7]. For example, experimental investigations on fire scenarios are not always straightforward to conduct owing to their consequences which can be devastating. As the case of Hasiba et al. [9] who used an experimental data to conduct a CFD parametric study for investigating the effect of the geometrical and thermal parameters of the heat source on the smoke spread inside a compartment fire. Another CFD study was carried out by Peppes et al. [10] for simulating the airflow outputted by a fire through a stairwell connecting two floors of an industrial building. Barozi et al. [8] studied the air movement in a 1:12 building model using a two-dimensional CFD model, and reported a good agreement between simulation and experimental results. Although CFD has turned out more popular in various areas 
with the development of computer ability and user-friendly CFD program interfaces (Tong et al. [11]; Tong and Zhang [12]). But the relative old computer methods are still used in this field and give satisfiable results. CFD simulation may not directly predict thermal load and energy consumption. Basically, decision-makers would need to compare the energy consumption of different design strategies. In order to esteem the energy reducing of the design, the application of energy simulation is very needful.

Among numerical approaches for the analysis of heat transfer. For example, discretization by using the finite-volume method which is, in this paper, used to study heat transfer cross four identical chimneys serving to exhaust the flue gases from the fireplaces of four contiguous apartments of one building. The simulations shown below have been performed using the CFD Fortran 90 coding.

\section{Problem Statement and Goals}

Fig. 1 represents the horizontal cross-section of this set of chimneys. The rectangular cross-section of each chimney has dimensions of $1,2 \mathrm{~m} \times 0,8 \mathrm{~m}$, in the outside, and $0,96 \mathrm{~m} \times 0,56 \mathrm{~m}$, in the inner hole. The chimney walls are made of firebrick, having an effective thermal conductivity $k=1.2 \mathrm{~W} / \mathrm{m}^{2} .{ }^{\circ} \mathrm{C}$.

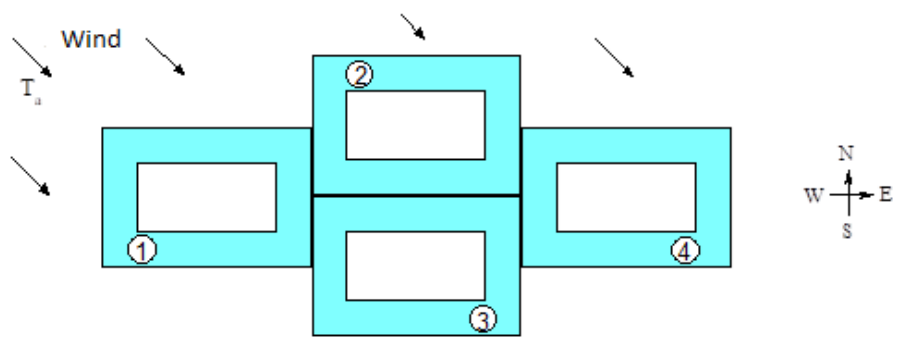

Fig. 1: The cross-section of the four chimneys.

Under the predominant wind direction in that region from Norwest, the heat transfer between the chimney outer surfaces and the ambient airflow (at $T_{a}=10^{\circ} \mathrm{C}$ ) is characterized by the values of the convective coefficient $h$ which is listed in Table 1:

Table 1: Average values of the convection heat transfer coefficient $h$ on the outer surface of each chimney wall, according to its orientation relatively to the wind.

\begin{tabular}{|c|c|c|c|c|}
\hline \multirow[b]{2}{*}{$\begin{array}{l}\text { Average convection } \\
\text { coefficient }\end{array}$} & \multicolumn{4}{|c|}{ Walls facing } \\
\hline & $\begin{array}{l}\text { Chimney } \\
1\end{array}$ & $\begin{array}{l}\text { Chimney } \\
2\end{array}$ & $\begin{array}{l}\text { Chimney } \\
3\end{array}$ & $\begin{array}{l}\text { Chimney } \\
4\end{array}$ \\
\hline$h_{1}=60 \mathrm{~W} / \mathrm{m}^{2} .{ }^{\circ} \mathrm{C}$ & $\mathrm{W}$ & $\mathrm{N}$ & - & - \\
\hline$h_{2}=h_{1} / 2$ & - & $E$ & - & $\mathrm{E}$ \\
\hline$h_{3}=h_{1} / 3$ & S & - & $\mathrm{W}, \mathrm{S}, \mathrm{E}$ & S \\
\hline$h_{4}=3 h_{1} / 4$ & $\mathrm{~N}$ & $\mathrm{~W}$ & - & $\mathrm{N}$ \\
\hline
\end{tabular}

When the respective fireplace is functioning, the flue gases rise through the hole at a temperature of $T_{f}=150^{\circ} \mathrm{C}$, exchanging heat with the inner surface of the chimney wall at a rate of. $h_{f}=50 \mathrm{~W} / \mathrm{m}^{2} .{ }^{\circ} \mathrm{C}$ Regarding the outer surfaces that are not exposed to the wind (walls in contact with a contiguous chimney), If the contiguous chimney is used, the heat transfer through the contacting walls will be practically negligible, therefore adiabatic conditions may be assumed.

Assuming that both data and conditions just described remain constant in time, It is determined the temperature distribution in the cross sections of chimneys number 1 and number 4 and calculated the rate of heat loss per unit height of each chimney, when the operating conditions are in case of all chimneys are used. The outer surfaces of chimney number 4 facing east and south absorb, respectively, 150 and $400 \mathrm{~W} / \mathrm{m}^{2}$ of the incident solar radiation. It is taken into account the heat losses by radiation from the outer surfaces (emissivity $\varepsilon=0.85$ ) exposed to the cold sky (supposed to be at $T_{s k y}=-20^{\circ} \mathrm{C}$ ). The density of firebrick $\rho=2.3 * 10^{3} \mathrm{~kg} / \mathrm{m}^{3}$ and the specific heat for it $c=1050 \mathrm{~J} / \mathrm{kg} .{ }^{\circ} \mathrm{C}$. 


\section{CFD Analysis Process \\ 3.1. Mathematical Model}

The general equation of conservation in Cartesian coordinates and tensor notation is:

$$
\frac{\partial(\rho \varphi)}{\partial t}+\frac{\partial}{\partial x_{j}}\left(\rho u_{j} \varphi-\Gamma_{\varphi} \frac{\partial \varphi}{\partial x_{j}}\right)=S_{\varphi}
$$

Where $\varphi$ can acquire different meanings: continuity $1, \mathrm{X}$ momentum $\mathrm{u}, \mathrm{Y}$ momentum $\mathrm{v}, \mathrm{Z}$ momentum w, and energy $\mathrm{h}$. In a preliminary analysis of the problem, the relevant variables in equation (1) have already been selected, resulting in a set of conservation equations to solve. The next step in the method of numerical integration of differential equations is called discretization, whose main function is transforming the differential (conservation) equations into algebraic equations (approximate, but resolvable) [2]. In this problem while the equation, which governs, is derived from the energy conservation equation and the problem can be considered two dimensional. Thus, from Equation (1) $\varphi=h, \Gamma_{\varphi}=k / c_{p}$, and $S_{\varphi}=S_{h}$. When we can assuming that the physical medium is incompressible then $h=c_{p} . T$ and the energy conservation equation sums up:

$$
\frac{\partial\left(\rho c_{p} T\right)}{\partial t}=\frac{\partial}{\partial x}\left(k \frac{\partial T}{\partial x}\right)+\frac{\partial}{\partial y}\left(k \frac{\partial T}{\partial y}\right)+S_{h} \quad\left[W / m^{3}\right]
$$

The conservation equation is integrated by applying a power balance to a small region of the domain (finite control volume) involving the mesh node. In this method, it is begun by dividing the computing domain (the domain is bordered by the outer surfaces of the chimney) into a number of contiguous control volumes such that there is a control volume involving each point of the mesh.

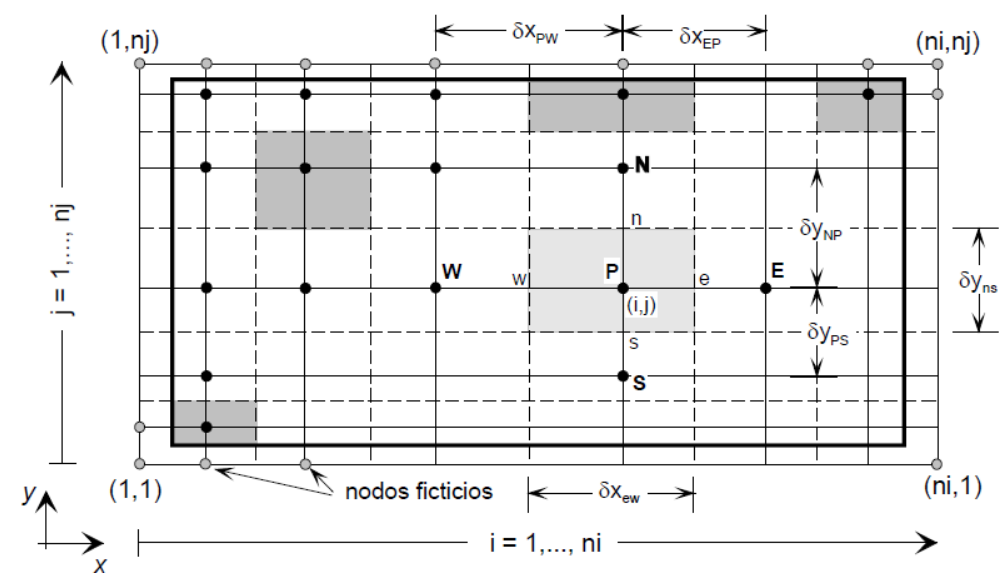

Fig. 2: Scheme of a mesh of nodes and associated finite volumes, in a system referenced in xy coordinates.

The algebraic (discretization) equation expresses the conservation principle of $T$ for the finite control volume, in the same way, that the original differential equation expresses it for an infinitesimal control volume. Fig. 2 shows a Cartesian mesh of nodes in the studied domain for a two-dimensional study, a finite-volume formulation.

Each node is associated with a control volume whose faces (the dashed line) are located mid-distance between neighbouring nodes. It is assumed that the values of $\mathrm{T}$ are located in the nodal positions. It should be noted that the boundaries of the domain of calculation, as well as of any obstacles or geometric singularities, coincide with the boundaries of the control volumes. For this purpose, the mesh generation includes outer lines of fictitious nodes, which are, in relation to the borders, in positions symmetrical of those of the adjacent internal nodes. A typical control volume within a computing domain, together with the respective node $\mathrm{P} \equiv(\mathrm{i}, \mathrm{j})$, the neighbors $(\mathrm{N}, \mathrm{S}, \mathrm{E}$ and $\mathrm{W})$ is shown in Fig. 3 and associated nomenclature. 
As a two-dimensional, in Cartesian coordinates $\mathrm{xy}(\mathrm{z})$, it is assumed that the control volume has unit depth $(\Delta z=1 \mathrm{~m})$, and its volume will be $\Delta V=S_{e w} \times S_{n s} \times 1$.

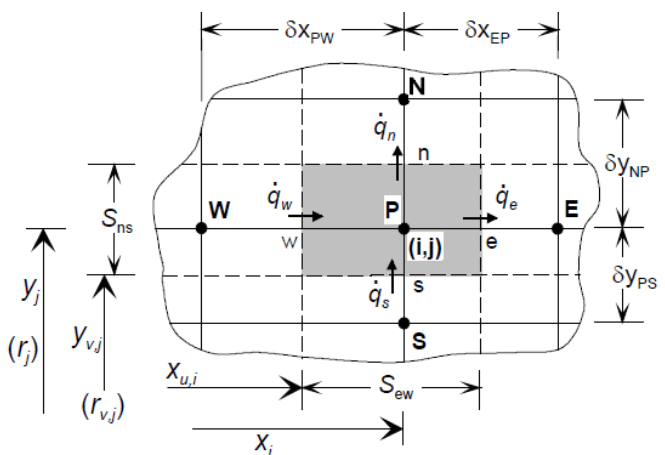

Fig. 3: Schematic of an interior generic node $\mathrm{P}$ of a Cartesian two-dimensional mesh (xy), together with the respective control volume. These faces are denoted by lowercase letters (n, s, e and w), in reference to the neighbouring nodes (N, S, E, and W) that communicate with P.

The discretization equation according to a totally implicit scheme can be written in its generic two-dimensional form:

$$
a_{P} T_{P}=a_{E} T_{E}+a_{W} T_{W}+a_{N} T_{N}+a_{S} T_{S}+b
$$

Where:

$$
\begin{gathered}
a_{E}=\frac{k_{e} \Delta y}{\delta x_{E P}}, a_{W}=\frac{k_{W} \Delta y}{\delta X_{P W}}, a_{N}=\frac{k_{n} \Delta x}{\delta y_{N P}}, a_{S}=\frac{k_{s} \Delta x}{\delta y_{P S}} \\
a_{P}^{0}=\frac{\rho c_{p} \Delta x \Delta y}{\Delta t} \\
b=S_{U} \Delta x \Delta y+a_{P}^{0} T_{P}^{0} \\
a_{P}=a_{E}+a_{W}+a_{N}+a_{S}+a_{P}^{0}-S_{P} \Delta x \Delta y
\end{gathered}
$$

The coefficients $\mathrm{a}_{\mathrm{E}}, \ldots, \mathrm{a}_{\mathrm{S}}$ represent the thermal conductance between the node $\mathrm{P}$ and its neighbours. The term $b$ results from the addition to the previous term of the rate of heat generation in the control volume associated with $S_{U}$ plus the internal energy contained in the control volume at time $t$. Finally, the central node coefficient, $a_{\mathrm{p}}$, is the sum of all neighboring coefficients, further containing a linearized thermo-source contribution.

The procedure for incorporating a boundary condition consists of two steps:

1. The coefficient $a_{\text {neighbor }}$ on the side of the boundary (cutting off the connection to it) is nullified.

2. The effect of the boundary on the adjacent control volume is introduced through additional source terms (which account for flows across the border).

In heat conduction situation, boundary conditions are divided for two types:

1. Frontier with known temperature. The usual method is to cut the "connection" of node $\mathrm{P}$ with node E, neutralizing the corresponding conductance $\mathrm{a}_{\mathrm{E}}=0$.

2. Known heat flux across the boundary (zero, if the boundary is adiabatic). The first step to incorporate this condition is to cut the node's connection to the boundary then canceling the corresponding coefficient. 
In convective boundary, the flow of heat across the boundary is not known at the outset. It is assumed that the boundary of the domain is exchanging heat with a fluid. Assuming the convection coefficient $h$ and the temperature of the fluid $T$ is known, the heat flow from the fluid is given by:

$$
\dot{q}_{\text {conv }, e}=h\left(T_{\infty}-T_{F}\right) \quad\left[W / m^{2}\right]
$$

It is possible to eliminate $T_{F}$ from equation (4), based on a thermal balance applied to a control surface coincident with the face and the control volume of $\mathrm{P}$ :

$$
\dot{q}_{c o n v, e}=\dot{q}_{e, P} \Rightarrow \frac{T_{\infty}-T_{F}}{1 / h}=\frac{T_{F}-T_{P}}{\delta X_{e P} / k_{P}}=\frac{T_{\infty}-T_{P}}{1 / h+\delta_{X_{e P}} / k_{P}}
$$

Thus, the thermal power entering the control volume of $\mathrm{P}$, through its face $\mathrm{e}$ (frontier) is:

$$
\dot{Q}_{c o n v, e}=\frac{T_{\infty}-T_{P}}{R_{\infty, P}}
$$

Where $R_{\infty, P}$ is the total heat resistance that the heat must "beat" in its path from the $\left(T_{\infty}\right)$ to the node P:

$$
R_{\infty, P}=\left(\frac{1}{h A_{e}}+\frac{\delta X_{e P}}{k_{P} A_{e}}\right) \quad\left[{ }^{o} C / W\right]
$$

Therefore the incorporation of this convective boundary condition requires the following modifications in the equation of the frontier node P: $a_{E}=0, S_{U}=S_{U}+\left(T_{\infty} / R_{\infty, P}\right)$, and $S_{P}=S_{P}-\left(1 / R_{\infty, P}\right)$.

\subsection{Solution Method}

The system of formally linear algebraic equations consisting of the discretization equations of all domain is solved by an iterative technique line-by-line, which is based on the so-called Thomas algorithm or TDMA 3 (tri-diagonal matrix algorithm) [1]. Considering the resolution of the equation of $\varphi$ along a column of $i$ of nodes (see Fig. 2), the discretization equation (3) can be written in the following form - to a node $(\mathrm{i}, \mathrm{j})$ any in that column and substituting $\mathrm{T}$ for $\varphi$, for convenience:

$$
D_{j} \varphi_{i, j}=A_{j} \varphi_{i, j+1}+B_{j} \varphi_{i, j-1}+C_{j}
$$

Where:

$$
A_{j}=a_{N}, \quad B_{j}=a_{S}, \quad D_{j}=a_{p}, \quad C_{j}=a_{E} \varphi_{i+1, j}+a_{W} \varphi_{i-1, j}+b
$$

The auxiliary coefficients $P_{j}$ and $Q_{j}$ are then defined:

$$
P_{j}=\frac{A_{j}}{D_{j}-B_{j} \cdot A_{j-1}}, \quad Q_{j}=\frac{C_{j}+B_{j} \times C_{j-1}}{D_{j}-B_{j} \cdot A_{j-1}}
$$

Which, after being calculated for the whole column in the "progressive" sense (increasing), allow the updated calculation of $\varphi_{i, j}$ throughout this column, through: 


$$
\varphi_{i, j}=P_{j} \varphi_{i, j+1}+Q_{j}
$$

by going in the "regressive" sense, from decreasing $j$ (from $j=n j-1$ to $j=2$ ).

This resolution procedure is extended to the entire domain, sweeping it, line-by-line. The calculation program called "COND-2D_Didact", which is used in this problem, was adapted for pedagogical purposes from a more complex TEACH program [Gosman (1975)][3,4]. It is intended to solve transient or stationary heat conduction problems in two-dimensional domains referable in cartesian or cylindrical coordinates, and governed by Equation (2). Where only the permanent regime (steady state) solution is concerned, it will be interesting to "simulate" a single "time jump", which can be achieved by specifying $\Delta t \rightarrow \infty \quad\left(\mathrm{eg}, \Delta t=10^{30} \mathrm{~s}\right)$ or by programming. The solution is then obtained through a succession of the iterative calculation cycles described above until the convergence criterion is reached.

\section{Results and Discussion}

Presentation of results (by using Tecplot software) is included graphs of the temperature distribution, as well as heat flow lines and vectors as it is shown in Fig. 4 for the chimney number 1 and Fig. 5 for the chimney number 4 . From Figs. 4 and 5, it can be recognized the effect of walls boundaries conditions on the predicted heat transfer. Where the heat flux through for the east wall of chimney 1 and west wall of chimney 4 equal to zero due to the adiabatic condition. While Figs. $(4, b)$ and $(5, b)$ show the differences in the heat flux distribution close to the other walls of each chimney due to the different conditions which are incident in the other directions.

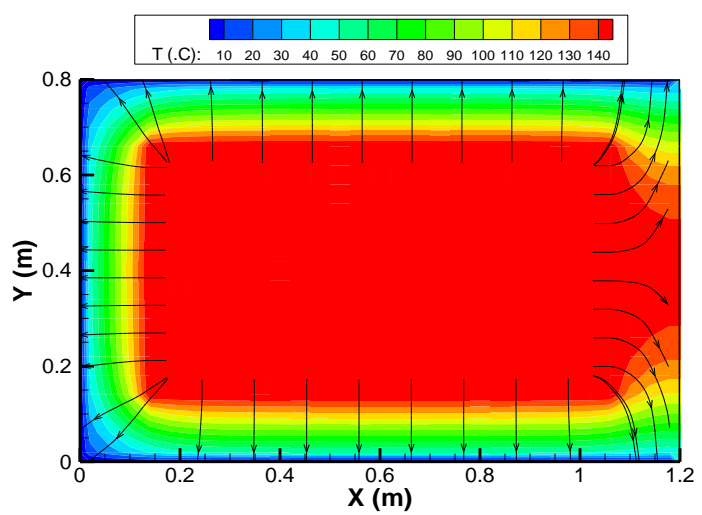

(a)

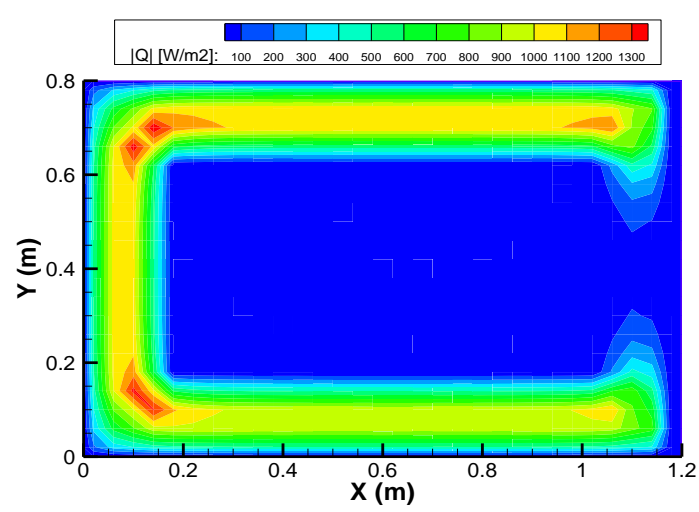

(b)

Fig. 4: Temperature distribution and heat flow lines along of the chimney number 1 (a) and heat flux distribution (b).

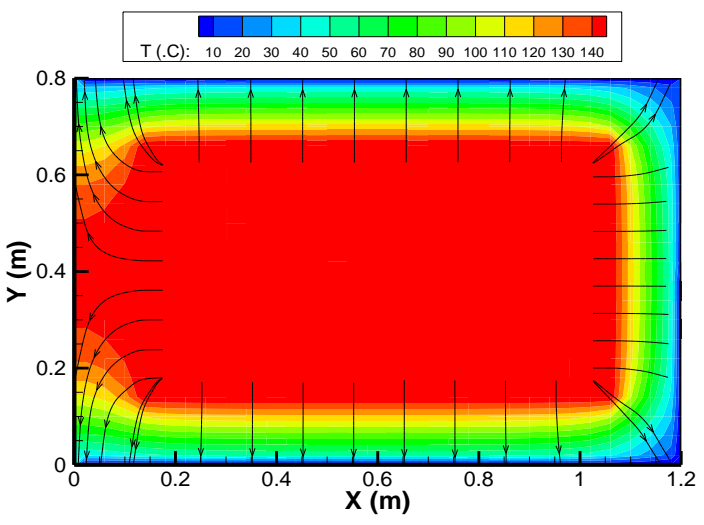

(a)

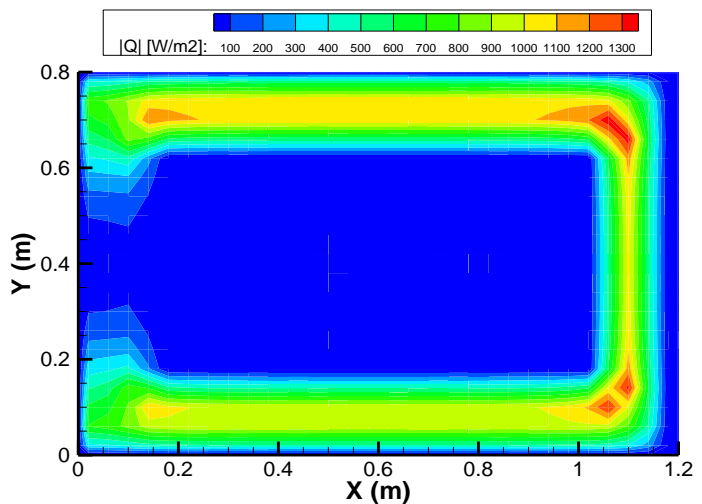

(b)

Fig. 5: Temperature distribution and heat flow lines along of the chimney number 4 (a) and heat flux distribution (b).

For each chimney (number 1 and 4) and by taking the coarsest mesh, it was made a comparison between the values of the overall heat loss through the chimney walls calculated from the numerical results at two different closed lines (close to 
the inner and outer surfaces). It was found that at these two lines the values of thermal flow are almost equal and this is evidence of the validity of the results. These values have reached for chimneys 1 and 4 to 2876 and $2871 \mathrm{~W} / \mathrm{m}$ respectively.

On the other hand, Investigation of the effect of the mesh size on the solution results is one of the steps performed in CFD study. Ideally, a mesh independent solution is one which does not change with further mesh refinement [6]. Grid sensitivity was carried out in both directions $(x, y)$ corresponding to $(32,22),(62,42),(122,82),(242,162)$ and $(482,322)$ grids, respectively. as it is shown in Fig. 6 by increasing the size of the grid which it is studied, heat loss reaches to values that the difference with the previous one has a small ratio. where at the grid of $(482,322)$ this difference reaches to less than $0.7 \%$ compared with the previous grid.

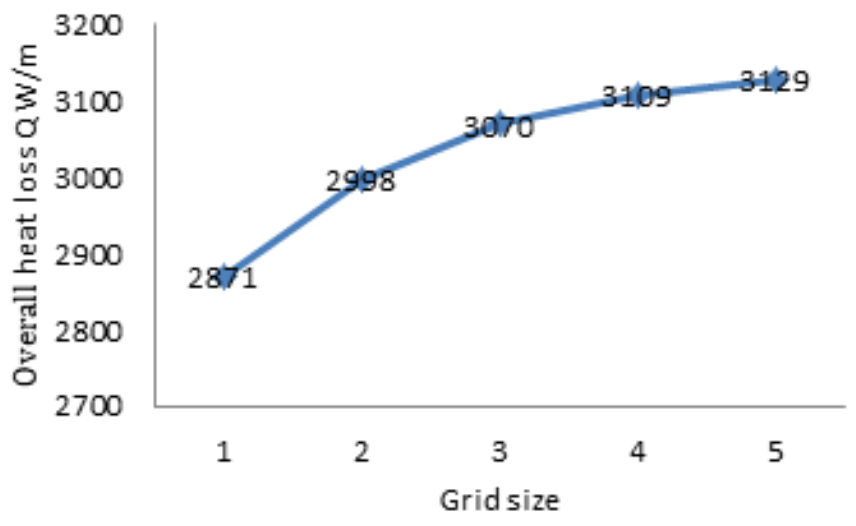

Fig. 6: The curve of comparison between the values of the Overall heat loss $\dot{Q}_{\text {tot }}[\mathrm{W} / \mathrm{m}]$ through the chimney 4 walls calculated from the numerical results at closed line close to the inner surfaces depending on different grid size (number of nodes in the study domain).

\section{Conclusion}

Simulations of heat transfer between the chimneys and with the natural boundary have been performed using an iterative technique line-by-line, which is based on the so-called Thomas algorithm or TDMA 3 (tri-diagonal matrix algorithm).

Presentation of results is included graphs of the temperature distribution, as well as heat flow lines and vectors. By taking the coarsest mesh, the overall heat loss through the chimney walls has reached for chimneys 1 and 4 to 2876 and 2871 $(\mathrm{W} / \mathrm{m})$ respectively. Where the results have referred to be care must be taken in a specification of walls boundary conditions, and especially the heat flows inside the boundary of the chimneys, and the situation of the functioning of contiguous chimneys which significantly influences the heat transfer coefficient profile interphase between the chimneys and the boundary of the domain which is the base of this study. The results of the comparison of the mesh sizes are close to the ideal situation which say that a mesh independent solution is one which does not change with further mesh refinement.

Thus this study has demonstrated that CFD is a powerful tool capable of capturing the complex heat transfer process (with some assumptions) in gas-solid structures with satisfactory results and is a useful tool in the design of other structures reactors. Where this investigation it can be used in order to achieve a recovering in energy systems run by ordinary chimneys.

\section{References}

[1] S. V. Patankar, Numerical Heat Transfer and Fluid Flow. Hemisphere Publishing Corporation, 1980.

[2] L. A. Oliveira, "Cálculo Numérico de Escoamentos com Transferência de Calor e de Mass," Dep. Engenharia Mecânica, FCTUC, 1989.

[3] A. D. Gosman, "A finite-difference calculation procedure for turbulent flows. I - Solution of equations for mean flow; II - Incorporation of two-equation turbulence models," lessons in. 8 and 9 of Turbulent course recirculating Flows, by A. D. Gosman, E. E. K. H. Khalil and B. E. Launder, Imperial College of Science and Technology, London, 1975.

[4] A. D. Gosman, "The TEACH-T computer program. I - Structure; II - Application," lessons in. 10 and 12 of the Turbulent Recirculating Flows course, by A. D. Gosman, E. E. K. H. Khalil and B. E. Launder, Imperial College of Science and Technology, London, 1975.

[5] A. Sharma, "Level set method for computational multi-fluid dynamics: A review on developments, applications, and analysis," Sadhana, Indian Academy of Sciences, vol. 40, no. 3, pp. 627-652, 2015. 
[6] M. Lungu, J. Sun, J. Wang, Z. Zhu and Y. Yan, “Computational fluid dynamics simulations of interphase heat transfer in a bubbling fluidized bed," Korean J. Chem. Eng., vol. 31, no. 7, pp. 1148-1161, 2014.

[7] A. Moftakhari and C. Ghazvin, "Natural element method study of combined natural convective and radiative heat transfer in irregular-shaped mediums with radiative properties," International Journal of Thermal Sciences, vol. 122 pp. 141 e 161, 2017.

[8] G. S. Barozzi, M. S. E. Imbabi, E. Nobile, and A. C. M. Sousa, Building Environ., 1992, vol. 27, no. 4,pp. $433-445$.

[9] R. Hasiba, R. Kumarb, S. Kumar, "Simulation of experimental compartment fire by CFD," Building and Environment, vol. 42, pp. 3149-3160, 2007.

[10] A. A. Peppes, M. Santamouris and D. N. Asimakopoulos, "Buoyancy driven flow through a stairwell," Building and Environment, vol. 36, pp. 167-180, 2001

[11] Z. Tong, Y. J. Wang, M. Patel, P. Kinney, S. Chrillrud and K.M. Zhang, "Modeling spatial variations of black carbon particles in an urban highway-building environment," Environmental Science \& Technology, vol. 46, pp. 312-319, 2012.

[12] Z. Tong, K. M. Zhang, "The near-source impacts of diesel backup generators in urban environments," Atmospheric Environment, vol. 109, pp. 262-271, 2015. 